\title{
Rursus
}

Russus

Poiétique, réception et réécriture des textes antiques

$1 \mid 2006$

Le modèle animal (I)

\section{L'animal et la pensée médicale dans les textes du Haut Moyen Age}

Jacques Voisenet

\section{OpenEdition}

Journals

Édition électronique

URL : http://journals.openedition.org/rursus/50

DOI : 10.4000/rursus.50

ISSN : 1951-669X

Éditeur

Université Nice-Sophia Antipolis

Référence électronique

Jacques Voisenet, «L'animal et la pensée médicale dans les textes du Haut Moyen Age », Rursus [En ligne], 1 | 2006, mis en ligne le 09 juillet 2006, consulté le 30 avril 2019. URL : http:// journals.openedition.org/rursus/50; DOI : 10.4000/rursus.50

Ce document a été généré automatiquement le 30 avril 2019

Rursus 


\title{
L'animal et la pensée médicale dans les textes du Haut Moyen Age
}

\author{
Jacques Voisenet
}

1 Depuis l'Antiquité, les médecins se sont penchés sur le monde animal parce qu'une grande partie des médications étaient constituées de fragments, d'extraits d'animaux ou d'animaux entiers. Même sans entrer dans la composition d'une potion ou d'un onguent, on croyait aussi que l'animal pouvait participer au processus de guérison en prenant sur lui le mal de la personne atteinte (fonction de bouc émissaire) ou bien de protéger les gens valides des assauts de maladie ou dangers, en raison de vertus qui lui étaient reconnues (fonction apotropaïque éloignant les influences maléfiques). Enfin, le comportement de l'animal qui sait, par une intelligence instinctive, trouver les remèdes ou les gestes qui favorisent sa guérison, sert d'exemple aux hommes. L'animal est donc omniprésent dans la littérature scientifique et médicale de l'Antiquité, en servant à la fois de remède, de support de la maladie, de protection contre elle ou de modèle pour suivre les gestes salvateurs.

2 Ces quelques pages ne prétendent pas dresser le tableau exhaustif des diverses fonctions curatives ou protectrices de la bête durant l'Antiquité et de leur diffusion au Moyen Age. Par exemple, la pharmacopée animale est extrêmement riche avec une multitude de recettes que l'on trouve dans des sources isolées mais qui, le plus souvent, se répètent d'ouvrages en ouvrages et traversent les siècles. Je me suis donc particulièrement intéressé à la transmission de ce savoir médical antique au Moyen Age, en privilégiant les premiers siècles - en gros du $\mathrm{V}^{\mathrm{e}}$ au XII ${ }^{\mathrm{e}}$ siècle. Dans un premier livre, Bestiaire chrétien. L'imagerie animale des auteurs du Haut Moyen Age ( $\mathrm{V}^{\mathrm{e}}-\mathrm{XI}{ }^{\mathrm{e}} \mathrm{s}$.), je me suis penché sur la récupération par les auteurs chrétiens des connaissances portant sur le monde animal en général.

3 Rappelons brièvement quelle a été l'attitude des hommes du Haut Moyen Age occidental face à la culture antique ${ }^{1}$. Malgré une certaine méfiance des clercs (qui entraîne le rejet de thèmes à la résonance trop païenne), l'Antiquité impose avec vigueur sa tutelle au Haut Moyen Age. Il faut distinguer les grecs des latins car ceux-ci ne sont souvent que des vulgarisateurs des connaissances sur le monde animal diffusées par les premiers 
(Aristote, Elien de Préneste, Dioscoride...). Le Moyen Age, jusqu'à une date tardive, n'utilise ce savoir que par l'intermédiaire de certains auteurs latins comme Pline (c'est ce qui explique la place privilégiée que nous lui est ici accordée). Il faut encore savoir que grecs et latins avaient des conceptions opposées de l'animal. Les premiers, à travers leurs philosophes, l'envisagent comme un inférieur ou comme un instrument (d'où son instrumentalisation dans les pratiques médicales) alors que les zoographes et les auteurs animaliers latins considèrent l'étude de l'animal comme "une variante, un duplicata, parfois même un modèle de l'éthique humaine ${ }^{2}$ ». Il s'agit d'une double vision, utilitaire et morale, que le christianisme médiéval a globalement repris en y ajoutant une dimension spirituelle.

\section{Les sources antiques de la pensée médicale médiévale}

4 Le Haut Moyen Age doit ses connaissances médicales concernant les animaux à quelques auteurs, finalement peu nombreux. Parmi les naturalistes deux se détachent: Aristote et Pline. Le premier (IV siècle avant J.-C.) a donné à la science antique une série d'ouvrages, consacrés en grande partie ou en totalité, à la faune. Par cette œuvre immense, Aristote s'est imposé comme le fondateur de la zoologie et de la zoohistoire et un maître incontesté jusqu'à Linné et Cuvier. Mais le Haut Moyen Age ne l'a connu qu'à travers Pline, avant sa redécouverte au XIII ${ }^{e}$ siècle par Albert le Grand ou Thomas d'Aquin grâce à la médiation d'Avicenne (980-1037, commentateur) et d'Averroès (1126-1198, adaptateur). Aristote a composé une véritable œuvre de science, remarquable par sa tentative de classification. Il manifeste souvent un authentique esprit critique dans son approche du monde animal, négligeant les bêtes merveilleuses et les récits fabuleux. Le Moyen Age lui est redevable d'une multitude de faits zoologiques et de descriptions anatomiques. Par contre, ses efforts pour aller du simple au général et tenter de dégager les lois et principes de la nature en s'appuyant sur une méthode scientifique (pas toujours rigoureuse il est vrai) n'ont pas été retenus. Les auteurs médiévaux ont souvent préféré les anecdotes fabuleuses et le bestiaire plus extraordinaire de Pline.

Pline l'Ancien ( $\mathrm{e}^{\mathrm{er}}$ siècle après J.-C.), surnommé le Naturaliste, laisse lui aussi une œuvre considérable constituée de 37 livres, son Histoire naturelle, dont nous retiendrons les livres VIII à XI pour le monde animal et XXVIII à XXXII tout particulièrement pour la pharmacopée animale. Largement tributaire d'Aristote, mais souvent mal restitué, il constitue une gigantesque compilation, sans méthode ni critique. Son Histoire naturelle abonde en croyances et récits légendaires, montrant bien son goût pour les anecdotes merveilleuses recueillies sans esprit critique. Il tire ses connaissances d'autorités médicales, des médecins comme Diodore, Nicandre ou Quintus Sextius Niger $^{3}$ ou des sages-femmes comme Olympias de Thèbes ${ }^{4}$ mais il laisse aussi une large place aux mages comme Osthanès, mages perses de Xerxès ou d'Alexandre. Ainsi pour lutter contre l'incontinence il conseille, à leur suite, de boire dans du vin doux de la cendre de verge d'âne et d'uriner dans la niche d'un chien en prononçant la formule : "C'est pour ne pas pisser au lit comme un chien dans sa niche ${ }^{5}$ ». Il a quand même, de temps en temps, quelques scrupules à raconter de telles balivernes. Il formule parfois certaines réserves mais pour finalement relater ces histoires extraordinaires. Il le fait justement à propos des médications animales : "Voilà ce que nous avons cru pouvoir rapporter, et encore, pour la plupart de ces recettes, non sans nous excuser de les produire; le reste n'est 
qu'abominations et infamies ${ }^{6}$ ». «Je ne doute pas en effet du mépris que montreront quelques uns pour ce que je dirai bientôt des animaux ${ }^{7}$ ». Il reconnaît que l'art médical, par la variété des traitements, change chaque jour et est entraîné «par le vent du charlatanisme de Grèce ${ }^{8}$ » parce que « la médecine est le seul art où l'on donne aussitôt sa confiance au premier venu se disant médecin alors qu'il n'en est point où l'imposture soit plus redoutable ${ }^{9}$. Peu importe le caractère extravagant des pratiques thérapeutiques et la composition répugnante des médications (urine d'animaux, fientes, sécrétions diverses : neuf grains de fiente de lièvre à prendre par les femmes qui veulent garder les seins droits ${ }^{10}$ ) puisque "c'est seulement chez le médecin que l'homicide est assuré de l'impunité totale ${ }^{11}$ ». Les auteurs du Moyen Age ont souvent repris à leur compte les récits de Pline mais en oubliant ses accès - modérés - de scepticisme. Il est l'auteur antique le plus cité par Isidore de Séville (vers 570-636) dans le livre XII, consacré aux animaux, de son ouvrage encyclopédique, Les étymologies, ou par Raban Maur dans son De universo d'époque carolingienne qui recopie lui-même Isidore. Celui-ci joue un rôle fondamental dans la transmission du savoir antique et en particulier de Pline, pas toujours utilisé directement mais souvent par l'intermédiaire d'abréviateurs. C'est le cas de Solin (III $\mathrm{s}$. après J.-C.) qui compile dans son recueil, Polyhistor, de nombreux récits extraordinaires empruntés à Pline, mais aussi à d'autres auteurs (Elien, Suétone...), qu'il simplifie pour ne conserver que le caractère merveilleux, état d'esprit qui annonce la mentalité médiévale. De même, les recettes médicales de Pline ont été réunies par un auteur anonyme du IV siècle dans l'ouvrage intitulé Medicina Plinii.

De toute façon, qu'il ait été connu directement (il existe plus de deux cent manuscrits médiévaux de l'Histoire naturelle) ou par l'intermédiaire d'abréviateurs comme Solin, Pline exerce un très fort ascendant sur toute la "science " médiévale. Celle-ci accède à travers lui au savoir des zoologistes grecs et reçoit en même temps tout un arsenal de légendes et croyances que les clercs mettent au service du christianisme et de sa vision morale et théologique. Le savoir animalier antique est donc passé au travers de filtres successifs (Solin, Isidore de Séville, Raban Maur...) provoquant un appauvrissement, une schématisation, voire une modification et il est parfois bien souvent difficile de reconnaître l'« origine » antique d'une pratique ou d'un récit, d'autant plus que les clercs ont cherché à "décontaminer » les histoires trop marquées par le paganisme. Cette réorientation de la matière animale antique est également perceptible pour la pharmacopée. Certes, la science médicale revêt un caractère beaucoup plus « neutre » que certains récits du merveilleux mythologique, et donc possède une grande facilité de récupération mais, cela se fait quand même au prix d'une adaptation, d'une christianisation d'une connaissance qui pouvait aussi bien servir à la guérison physique des hommes qu'à leur salut spirituel. Le merveilleux zoologique antique a été globalement reçu sans difficulté car il n'était pas considéré par les clercs comme nocif mais au contraire utile à leur propos.

7 De multiples recettes médicales ont ainsi été fournies, de façon directe ou indirecte, par les traités de zoologie comme l'Histoire naturelle de Pline, l'Histoire des animaux d'Aristote, le Corpus Hippocraticum ou encore De la nature des animaux d'Elien de Préneste (vers 170-v. 235). Cet italien, composant en grec, s'intéresse à la zoologie mais il intègre à cette discipline des histoires extraordinaires et des légendes, contrairement à l'école aristotélicienne qui avait repoussé le merveilleux et tenté de classer avec logique et d'expliquer rationnellement les faits. Il recommande par exemple aux voyageurs qui veulent traverser le désert de Libye d'emporter un coq pour se prémunir des attaques du 
terrible basilic, le roi des serpents ${ }^{12}$, recommandation faite également aux femmes enceintes qui faciliteront, grâce au coq, leur accouchement ${ }^{13}$...

Les traités proprement médicaux et pharmaceutiques accordent une part encore plus grande à aux médications animales. Nicandre de Colophon, grec de la fin du $\mathrm{III}^{\mathrm{e}} \mathrm{s}$. avant J.C. et auteur des Thèriaka qui traitent en environ un millier de vers des morsures des bêtes et de leurs remèdes (commenté sous le même titre - Thèriaka - au XII siècle par le byzantin Jean Tzetzès) ainsi que d'un autre ouvrage de 600 vers les Alexipharmaka sur les contrepoisons, souvent présentés comme les premiers balbutiements de la chimie avec une application pratique: la fabrication de poisons et de contrepoisons! Citons également le De materia medica (La matière médicale) de Dioscoride d'Anazarbe, médecin grec du I $\mathrm{I}^{\mathrm{er}} \mathrm{s}$. après J.-C qui offre en six livres une considérable nomenclature de plantes, résumant l'essentiel des connaissances botaniques antiques, mais aussi une centaine d'animaux à valeur thérapeutique. Il fût largement utilisé au Moyen Age, en particulier par les byzantins, mais aussi au-delà comme par le naturaliste de la Renaissance, Conrad Gesner, auteur d'un monumental traité de zoologie, Historia animalium. Enfin, toujours dans ces traités médicaux, rappelons la Medicina Plinii, déjà cité, du IV siècle.

\section{La transmission du savoir animalier}

Les recettes thérapeutiques ou prophylactiques de Pline sont largement réutilisées durant le Haut Moyen Age.

Pour les soins contre les morsures de serpents il évoque le cerf. «Personne n'ignore que les cerfs détruisent ces animaux et que, là où il s'en trouve, ils les tirent de leurs trous pour les manger. Or ce ne sont pas seulement les cerfs vivants qui combattent les serpents, mais aussi chacune de leurs parties prises à part. L'odeur de leurs cornes brûlées met, comme on l'a dit, ces reptiles en fuite; on prétend d'autre part qu'ils se rassemblent lorsqu'on fait brûler les os du haut de la trachée du cerf. Se coucher sur des peaux de cerf procure un sommeil à l'abri de leurs attaques ; la présure du cerf prise dans du vinaigre guérit leurs morsures, et l'avoir seulement maniée en garantit pour la journée (...). Les serpents fuient même ceux qui portent seulement une dent de cet animal ou qui sont frottés de moelle ou de suif soit de cerf, soit de faon. Mais aux meilleurs remèdes on préfère la caillette d'un faon extrait du ventre de sa mère. On dit que le sang du cerf, brûlé sur du bois de lentisque en même temps que de la serpentaire, de la sarriette et de l'orcanette, rassemble les serpents ; mais ils se dispersent ensuite si, après avoir ôté ce sang, on ajoute du pyrèthre ${ }^{14} »$. Au $\mathrm{VI}^{\mathrm{e}}$ siècle, Isidore de Séville rappelle cette inimitié : « Ennemis des serpents, quand ils se sentent incommodés par la maladie, ils les font sortir de leurs trous en soufflant par leurs naseaux et, sans souffrir de leur venin mortel, ils se guérissent en les dévorant ${ }^{15} »$. Isidore reste assez laconique en se contentant de citer la principale particularité des cerfs que d'autres exploitent dans une optique toute autre. La capacité à se guérir lui-même et la clairvoyance du cerf ont alors servi le propos des clercs. Cette lutte revêt alors une véritable dimension salutaire, incarnant le combat du bien et du mal et montrant au croyant comment se guérir du mal suprême qu'est le péché. Le Physiologus, texte alexandrin du $\mathrm{II}^{\mathrm{e}}$ siècle qui a connu un énorme succès au Moyen Age, présente les animaux sous trois plans : réel, allégorique et moral. Cette hostilité est montrée comme celle qui oppose le Christ-cerf au diable-serpent, modifiant le rôle de l'eau qui ne sert plus à étancher la soif du cervidé mais à faire sortir le reptile de son trou : "Il emplit sa bouche d'eau à la source et la rejette dans les fissures 
de la terre où se cache le serpent, il le fait sortir ; il le foule aux pieds et le tue. De la même façon Notre Seigneur extermina le grand diable serpent par l'eau céleste" ${ }^{16}$. De même pour Raban Maur, le cerf «aspire les serpents par les naseaux; quand il les a dévoré, ébranlé par le venin bouillonnant, il se dépêche aussi vite qu'il le peut vers une source (... ). La belle comparaison entre le cerf et notre soif ardente nous pousse, lorsque nous aspirons le venin de l'antique serpent et que notre gorge brûle profondément, à nous hâter à la source de la divine miséricorde (...). Le cerf est, comme nous l'avons dit, vorace de serpents venimeux (...). C'est à bon escient qu'on le compare aux fidèles qui dévorent le diable quand ils changent la débauche en louanges et gloire du Seigneur $\gg .{ }^{17}$ Soutenu par l'imagerie biblique du cerf altéré qui recherche les sources ${ }^{18}$, l'animal devient donc pour le Moyen Age la figure du Christ, des apôtres, des prêtres ou des croyants qui luttent contre leurs propres vices ou contre Satan ${ }^{19}$.

11 Animal salutaire, il sert alors, en conformité avec les recettes de Pline, à réaliser de multiples amulettes faites avec ses dents, ses os, sa corne, son cuir ou ses poils. Pour lutter contre les morsures on composait aussi des mélanges de graisse, de sang ou de moelle avec de la sarriette ou de l'estragon ${ }^{20}$. Ces pratiques magico-médicales de l'Antiquité se maintiennent donc au Moyen Age tout en se christianisant. Elles préservent ceux qui y ont recours aussi bien du venin des serpents, de la mort physique que de la mort de l'âme. Pline recommandait de dormir sur une peau de cerf pour se prémunir des serpents, certains clercs se faisaient ensevelir dans une peau de cervidé pour assurer, par delà la mort, plus que la protection de leur dépouille, le salut de leur âme. Ainsi au XII ${ }^{\mathrm{e}}$ siècle, Roger de Beaufort, le futur pape Clément VI, demande à ce que son cadavre soit cousu dans une peau de cerf ${ }^{21}$. Les nombreux bijoux, bagues et fibules médiévales portant une image de cerf, se chargeaient probablement d'une fonction protectrice et apotropaïque (les clercs réclament fréquemment à ce qu'on remplace sur les vêtements et les bijoux les figures animales trop païennes - le serpent en particulier - par le cerf, la colombe, le poisson...). L'abbesse Hildegarde de Bingen (1098-1179), que l'on peut considérer comme la première zoologue du Moyen Age chrétien ${ }^{22}$ grâce à une observation véritable du monde animal dans les quatre livres de sa Physica, continue à véhiculer les remèdes de bonne femme rassemblés par Pline. Dans le Livre des subtilités des créatures divines, elle reprend le conseil de Pline sur les cornes de cerf brûlées : « Râpe la corne du cerf, ajoute de l'encens à ce que tu as râpé et brûle le tout : l'odeur chasse les esprits de l'air par la puissance que détiennent les cornes, tient en respect envoûtements et sorts, et chasse les mauvaises vermines ${ }^{23}$ ". Mais si ces remèdes, en particulier contre les morsures de serpents, se sont perpétués au Moyen Age, c'est parce que le cerf avait, dans le monde chrétien, une connotation symbolique positive. Les Psaumes louent sa course et sa fuite salutaires, sa recherche des lieux élevés, autant de comportements qui en font l'emblème du croyant prudent et avisé qui fuit le monde et ses tentations. Le cerf a donc continué à jouer un rôle éminemment protecteur, comme précieux instrument au service de la médecine des corps et de la médecine des âmes. La démarche curative lie de façon indissociable le plan physique et la sphère spirituelle. C'est parce qu'il y a eu conjonction, d'une part, entre la tradition scientifique antique et certaines croyances folkloriques et, d'autre part, l'imagerie biblique, que le cerf a pu ainsi maintenir sa position dans l'arsenal médical du Haut Moyen Age. D'autres animaux (porc, bouc, âne...), que Pline évoquait aussi comme des remèdes aux morsures de serpents, n'ont pas eu le même traitement ni la même postérité. Ainsi, il recommandait l'utilisation du foie ou de la cervelle de verrat macérée dans du vin ou du poumon d'âne en fumigation ${ }^{24}$. Mais ces bêtes n'ont pas bonne presse chez les clercs et leur connotation négative à cause de leur saleté ou de leur 
lubricité ne pouvait les intégrer à la cohorte des animaux prophylactiques. Malgré son humilité, vertu si chère aux clercs du Moyen Age, et le fait d'avoir été la monture du Christ pour entrer à Jérusalem, l'âne traîne une réputation de luxure. Grégoire le Grand affirme que le Démon « lorsqu'il incite à la luxure est un âne ». Sa cervelle était justement consommée comme un aphrodisiaque.

Pline précise également qu'il suffisait de murmurer à l'oreille de l'âne qu'on avait été piqué par un scorpion pour que le mal passe aussitôt à l'animal ! Cette capacité à absorber le mal se retrouve aussi au Moyen Age mais dans une perspective spirituelle. Si je n'ai pas trouvé de cas de transfert de venin de l'homme à l'âne, celui-ci remplit parfois le rôle de bouc émissaire se chargeant du mal d'autrui. Pour les maladies lunatiques, Hildegarde de Bingen conseille « de rechercher un endroit où l'on tue un âne ou bien il meurt tout seul, ou encore il se roule sur le sol: on fera ensuite coucher le malade sur le sol pendant un petit moment, caché par une couverture, il dormira, s'il peut ; puis on prendra sa main et on dira : «Lazare a dormi et s'est reposé, puis il s'est relevé; et, tout comme il a été arraché par le Christ à sa puanteur puante, toi aussi relève-toi de cette maladie dangereuse et de ces fièvres changeantes, toi qui te trouves dans la situation où le Christ s'est trouvé en s'asseyant sur un support de cette espèce [l'entrée à Jérusalem sur un âne], signifiant ainsi qu'il rachèterait l'homme de ses péchés et le redresserait ». Un petit moment après recommence au même endroit, par trois fois ; puis trois fois le lendemain ou le surlendemain; puis trois fois encore le lendemain ou le surlendemain, et il sera guéri $» .^{25}$ Dans la vie de saint Rieule, deuxième évêque d'Arles mort au III $^{\mathrm{e}}$ siècle, de rédaction postérieure au Haut Moyen Age, l'évêque libère un possédé en chassant l'esprit mauvais qui l'habitait. Celui-ci demande alors de pouvoir investir l'âne qui servait de monture à l'évêque, rappelant l'épisode néotestamentaire où des esprits prennent possession des porcs de Gérasa avant de se jeter dans la mer ${ }^{26}$. Mais l'animal, faisant preuve d'une étonnante clairvoyance, refuse de se laisser investir en traçant un signe de croix avec sa patte avant ${ }^{27}$. La guérison du possédé ne doit pas se faire au détriment de l'humble monture de l'évêque et l'âne, réputé pour sa sottise, donne ici une leçon au croyant en montrant que le meilleur moyen de s'opposer au mal est de faire un signe de croix.

Durant les premiers siècles du Moyen Age les clercs ont eu, dans la pratique quotidienne, beaucoup de mal à s'opposer aux méthodes curatives païennes proposées contre les morsures de serpents. Ils tentent d'offrir un protocole entièrement chrétien qui consiste à invoquer l'intercession de saints guérisseurs de morsures de serpent, à se rendre à leur tombeau (Grégoire de Tours souligne au $\mathrm{VI}^{\mathrm{e}}$ siècle ces guérisons se produisant sur la tombe de saint Phocas ${ }^{28}$ ) ou à recevoir la bénédiction d'un moine. La Vie des Pères du Jura $\mathrm{du} \mathrm{VI}^{\mathrm{e}}$ siècle rapporte que les moines anciens avaient ce pouvoir: "Le bienheureux Romain ne brillait pas seul au monastère par ces dons miraculeux : grâce à ce modèle de perfection et de charité, tous les frères, dans les merveilles qu'ils accomplissaient, suivaient l'exemple qu'il offrait à chacun. Ainsi, souvent, on voyait là beaucoup de personnes délivrées du venin des serpents ou débarrassées des troupes des démons ${ }^{29}$ ". Cette faculté de guérir n'est pas exclusivement réservée au saint - sa rareté et l'éloignement de son tombeau réduisaient d'autant la possibilité d'avoir un recours efficace - mais elle est étendue à tous les hommes d'Eglise. On tente de substituer au pouvoir du mage celui du clerc mais la concurrence païenne des charmes et formules magiques est encore très forte durant le Haut Moyen Age. Dans la vie de saint Eparchius, rédigée à l'époque carolingienne, l'hagiographe prend le soin de mentionner que le saint, 
mordu par un reptile, n'avait pas cherché à avoir recours aux incantations (carmina) pour se soigner, fait suffisamment exceptionnel pour être signalé et qui distingue le saint du vulgum pecus ${ }^{30}$. La prière est présentée comme une alternative efficace aux formules magiques et les vitae des saints multiplient à partir du XII ${ }^{\mathrm{e}}$ siècle les exemples de guérison grâce à l'intervention du saint.

Mais souvent, les clercs, devant la pression de ces pratiques anciennes, se contentent souvent de les christianiser en changeant les formules, en ajoutant des ingrédients chrétiens (l'encens mêlé par Hildegarde à la corne de cerf brûlée) ou des gestes salutaires (le signe de croix) qui renforcent et cautionnent l'utilisation de l'animal et de ses propres vertus reconnues depuis des siècles.

\section{L'adaptation des recettes : le cas de la sexualité}

\section{Remèdes et sexualité}

\begin{tabular}{|c|c|c|}
\hline \multirow[t]{8}{*}{$\begin{array}{l}\text { Stimulation des fonctions } \\
\text { sexuelles }\end{array}$} & Moelle de porc & \multirow[t]{8}{*}{$\begin{array}{l}\text { HN XXVIII, } \\
80\end{array}$} \\
\hline & Suif d'âne et graisse de jars en onction & \\
\hline & Humeur de jument s'écoulant après la saillie & \\
\hline & Testicules desséchés de cheval en breuvage & \\
\hline & Testicule droit d'un âne et vin & \\
\hline & $\begin{array}{l}\text { Ecume d'âne après saillie sur une étofe rousse placée dans un } \\
\text { médaillon d'argent }\end{array}$ & \\
\hline & $\begin{array}{l}\text { Plonger sept fois une verge d'âne dans de l'huile bouillante, } \\
\text { puis friction sur l'organe défaillant }\end{array}$ & \\
\hline & Urine de taureau qui vient de saillir & \\
\hline
\end{tabular}

Chez Pline, les médications destinées à stimuler les fonctions sexuelles s'appuient sur des animaux dont la nature se caractérise par la vigueur sexuelle (l'âne, le jars, le cheval, le taureau...). La réputation de ces animaux a largement été développée par les auteurs de l'Antiquité. L'âne, par la taille de son sexe et son penchant pour l'accouplement incarne les plaisirs charnels. Dans les Métamorphoses d'Apulée, Lucius se transforme en âne après avoir fréquenté une courtisane. Le jars, oiseau au comportement sexuel remarquable, est associé à Aphrodite et à son fils Priape auquel on sacrifiait des ânes. Sa fougue le classe parmi les animaux chauds et le pousse à rechercher le froid. Ainsi pour Elien : «Comme le jars est de nature ardente et qu'il s'enflamme, il aime nager, il aime les aliments très froids, l'herbe, la laitue et autres nourritures qui engendrent le froid ${ }^{31}$ ». La jument et le cheval manifestent un désir particulier à l'accouplement. Pour Aristote : «De toutes les femelles, la plus ardente à désirer l'union est la jument; ensuite vient la vache. Les juments ont la folie du mâle: de la vient que, en guise d'insulte, le nom de cet animal et lui seul, est appliqué à la femme qui s'abandonne sans retenue aux plaisirs de l'amour. (...) 
Quand les cavales sont en état de rut (...) elles laissent s'écouler une certaine humeur appelée hippomane, (...) elle est recherchée entre toutes par les femmes dans la préparation des breuvages ${ }^{32} »$. Toujours pour Aristote, la jument est la seule femelle, avec la femme, à accepter l'accouplement lorsqu'elle est gravide ${ }^{33}$. Quant au cheval il évoque aussi fortement la sexualité. La figure de l'étalon hennissant après la jument est un lieu commun littéraire et symbolise les désirs effrénés de l'homme (Plaute, Cicéron, Apulée, Ovide, Claudien... $\left.{ }^{34}\right)$. Il était donc normal que ces animaux réputés pour l'ardeur de leur désir et la force de leur accouplement entrent dans la composition de potions aphrodisiaques ou de rites magiques (le contact de la verge d'âne). Ces recettes et pratiques ont continué à exister au Haut Moyen Age mais de façon occulte. La culture savante, aux mains des clercs, se garde bien de véhiculer ces croyances à propos des aphrodisiaques d'origine animale et n'en parle que pour les dénoncer. Il y a donc une certaine rareté des témoignages en ce domaine. La répression de l'Eglise a été particulièrement forte durant les premiers siècles du Moyen Age où se met en place un véritable christianisme de combat qui cherche à éradiquer les aspects les plus criants de la culture païenne. Le concile de Tours de 813 condamne ainsi le port des ossements d'animaux: "Que les prêtres avertissent leurs fidèles: ils doivent savoir que les incantations et la magie ne peuvent rien contre les infirmités humaines ni les faiblesses des animaux, ni leur claudication ni même leurs maladies ; les amulettes d'os ou d'herbes qu'arborent les mortels ne sont pas utiles. Ce sont les lacs et les pièges que l'antique ennemi emploie pour tenter d'attraper, ce perfide, le genre humain ${ }^{35} »$. Un des rares moments où ces pratiques apparaissent se produit dans les ouvrages répressifs destinés aux confesseurs qu'il faut instruire des pratiques illicites de leurs ouailles et de la pénitence qu'il doit leur infliger. Ces manuels de confesseur qui évoquent toutes les fautes et péchés possibles portent le nom de Pénitentiels et ont connu une grande vogue au Haut Moyen Age. C'est le cas de celui de l'évêque de Worms en l'an Mille, Burchard, dont l'ouvrage pénitentiel porte le nom évocateur de Corrector (il est destiné à corriger) ou de Medicus (véritable médecin des âmes) ${ }^{36}$. L'évêque signale le comportement peccamineux de femmes qui cherchent à redonner de la vigueur sexuelle à leur époux. Elles s'introduisent un poisson vivant dans le vagin jusqu'à ce qu'il étouffe et elles le donnent ensuite à manger à leur mari défaillant. Ces femmes encourent une sanction de deux ans de jeûne ${ }^{37}$. Le poisson, en tant que figure de la fécondité et de la vie bénéficiant d'une tradition fort répandue et ancienne, sert dans ce cas de véritable substitut phallique que les clercs se devaient de dénoncer. Mais le poisson, malgré ces pratiques de magie populaire, est sauvé par sa nature christique et baptismale qui le maintient dans la cohorte des « bons » animaux.

Quant à ceux qui entraient dans la composition des philtres amoureux de Pline (âne, cheval, taureau...), les auteurs du Moyen Age ne remettent pas en cause leur nature fortement sexuée. Au contraire ils l'utilisent pour dénoncer les excès de la sexualité et la recherche du plaisir. L'âne, l'étalon hennissant, le taureau vigoureux symbolisent le péché de chair, les fornicateurs, les adultères puisqu'ils s'accouplent entre espèces différentes, l'âne avec la jument et le cheval avec l'ânesse, donnant naissance à des individus hybrides, le mulet et le bardot, fortement suspects aux yeux des clercs à cause de leur double nature. Ils représentent donc le péché, véritable obstacle au salut, et annoncent la mort de l'âme. Ils ne peuvent donc être intégré à une démarche curative et il faut manger leur chair avec prudence. La diététique médiévale insiste sur la nature particulière de ces viandes. Ainsi la chair de l'âne, animal chaud et rendu stupide " par la 
surabondance de forces dont il dispose pour la fornication », (...) «n'est pas bonne à manger pour l'homme, car elle est souillée à cause de la stupidité qui est en lui ${ }^{38}$ ». La viande de porc doit être aussi évitée car « le porc est chaud, il a une nature ardente ; (...) il est glouton, toujours avide de manger, au point de ne pas faire attention à ce qu'il mange, si bien qu'il mange parfois des aliments immondes. (...) C'est un animal impur, sa chair n'est pas pure, mais de valeur douteuse; elle n'est bonne à manger ni pour les bienportants ni pour les malades, car elle ne diminue en l'homme ni le flegme ${ }^{39} \mathrm{ni}$ les autres maladies, mais les augmente plutôt : en effet, sa chaleur s'ajoute à celle de l'homme et soulève dans son comportement et ses actions des tempêtes qui sont des malheurs pour l'homme ${ }^{40} »$. Consommée avec modération ou appliquée en onguent, la viande de porc (parfois sa peau) peut quand même guérir de la lèpre suivant le principe de soigner le mal (une maladie particulièrement impure) par le mal (une viande impure) ${ }^{41}$. Les viandes du porc «excitent facilement chez les hommes le goût du plaisii ${ }^{42}$ ». On les recommande malgré tout pour lutter contre la stérilité : « L'homme dont la semence est de nature trop fluide, si bien qu'elle n'est pas fertile, prendra des chatons de noisetier, un tiers de joubarbe, puis du liseron et un peu de poivre commun : il fera cuire cela avec le foie d'un jeune bouc déjà apte à la reproduction, en y ajoutant un peu de chair de porc, crue et grass $^{43}$ ». Les animaux fortement sexués (le bouc et le porc) ne sont plus réquisitionnés dans une démarche qui valorise le plaisir (la composition d'aphrodisiaques) mais dans le traitement de la stérilité qui est un obstacle à la recommandation divine : "Croissez et multipliez ». Malgré le maintien de ces animaux dans une perspective médicale, on mesure ici la réorientation de la pratique sexuelle qui n'est plus placée sous le signe du plaisir mais de la procréation. Cette élite intellectuelle, composée essentiellement de clercs (abbesse comme Hildegarde, moine, prêtre, évêque...), puise largement dans l'arsenal médical antique mais en lui donnant un éclairage nouveau, en fonction de ses préoccupations (chasteté, lutte contre le péché) et de ses phobies (la sexualité). Puisque la viande c'est de la chair, et la chair le plaisir, il faut pour les clercs adopter un régime végétarien, et pour les laïcs modérer et surveiller leur consommation de viande. Le remède contre la luxure est de supprimer les viandes qui échauffent et de prendre une potion végétale. "Pour que l'homme éteigne en lui le goût du plaisir et l'amour de la chair, il lui faut recueillir en été de l'aneth, deux fois autant de la menthe d'eau, de la pulmonaire, (...) de la racine d'iris d'Illyrie, (...) de l'ail d'Ascalon (...) ; qu'il mette le tout dans du vinaigre et qu'il en fasse un condiment. (...) Le sec et le froid de l'aneth éteignent la chaleur du plaisir, le suc froid de la menthe d'eau résiste à son suc dépravé, le suc froid et peu agréable de la pulmonaire enlève le charme de la dépravation, le froid vertueux de l'iris d'Illyrie domine le goût du plaisir et le froid vénéneux de l'ail fait diminuer le poison pervers du plaisir ${ }^{44} »$. La théorie physique des quatre éléments, fondée sur les qualités opposées du chaud et du froid, de l'humide et du sec, est ainsi mise au service d'une morale sexuelle rigoureuse.

La chair du cheval est aussi jugée « dure, difficile à manger, mauvaise pour l'homme, tant et si bien qu'il a beaucoup de peine à la digérer ${ }^{45} »$. Elle est donc peu recommandable d'un point de vue diététique - elle échauffe trop - mais surtout parce qu'elle est liée à des pratiques païennes en vigueur dans les populations germaniques. Elles attribuaient à la viande des chevaux sacrifiés (souvent en l'honneur des défunts) une vertu phylactérique. Cette manducation permettait de participer à la nature magique de l'animal et d'établir un lien entre le monde des vivants et celui des morts. L'hippophagie ${ }^{46}$ et l'absorption de sang étaient une pratique largement répandue chez les barbares. Pline avait déjà évoqué la coutume des Sarmates qui prélevaient du sang à leurs chevaux pour se nourrir ${ }^{47}$. Pour 
les auteurs chrétiens, il ne s'agit pas d'une habitude alimentaire innocente mais d'un mode de vie païen qu'il faut éradiquer. Saint Boniface (mort en 755), apôtre de la Germanie, reçoit des recommandations très strictes de la part du pape Grégoire III (731-741) pour qui il faut interdire aux nouveaux convertis la consommation de viande de cheval, sauvage ou domestique, et imposer une sanction sévère aux contrevenants car c'est un usage " immonde et exécrable ${ }^{48} »$. Vingt ans plus tard, le pape Zacharie (741-752) lui renouvelle l'interdiction à partir d'une liste d'animaux que Boniface lui avait fait parvenir ${ }^{49}$. Cela montre la difficulté que l'évangélisateur devait rencontrer à faire accepter cette interdiction par les populations germaniques habituées à manger de la viande de cheval. Les besoins d'éclaircissements de Boniface étaient d'autant plus forts qu'il savait que dans la chrétienté cette sévérité ne faisait pas l'unanimité. Certains pénitentiels se montrent intransigeants (trois à quatre ans de pénitence pour les mangeurs de chevaux), d'autres sont plus mesurés: «La consommation du cheval n'est pas interdite, pourtant elle n'est pas habituell ${ }^{50}$ »; «La viande chevaline n'est pas interdite même si beaucoup de peuples refusent d'en manger ${ }^{51}$ ». C'est le cas en particulier des orientaux ou des irlandais mais l'interdiction stricte des papes Grégoire et Zacharie est imposée dans un contexte d'évangélisation sur un front du christianisme et à cause de la place du cheval dans la religion germanique. Manger n'est pas un geste anodin, cela peut vous maintenir dans l'erreur du péché, maladie spirituelle qui a toujours une incidence sur le corps et provoque les maladies physiques ${ }^{52}$.

Ces quelques exemples autour des viandes d'âne, de porc ou de cheval montrent que la consommation de l'animal n'est pas seulement perçue d'un point de vue diététique ou médical mais qu'elle est sous-tendue par de nombreuses notions et oppositions : chrétienpaïen, sain-malsain, pur-impur, licite-illicite. La médecine, comme le déplorait déjà Pline, reste au Moyen Age en étroite relation avec la magie et la religion. Hildegarde ne cesse de répéter à la fin de ses recettes médicales que si elles échouent « c'est (que) Dieu ne le veut $\operatorname{pas}^{53} »$.

\section{Le rejet}

HYĖNE

\begin{tabular}{|l|l|l|}
\hline Peau & Protection contre les panthères, la céphalée & $\begin{array}{l}\text { XXVIII, } \\
27\end{array}$ \\
\hline $\begin{array}{l}\text { Fiel avec miel et } \\
\text { safran }\end{array}$ & Cataracte, yeux chassieux & $\begin{array}{l}\text { XXVIII, } \\
27\end{array}$ \\
\hline Dent portée par un fil & Terreurs nocturnes & $\begin{array}{l}\text { XXVIII, } \\
27\end{array}$ \\
\hline Dents en application & Douleurs de dents, épaules et muscles des bras & $\begin{array}{l}\text { XXVIII, } \\
27\end{array}$ \\
\hline $\begin{array}{l}\text { Oeil avec réglisse et } \\
\text { aneth }\end{array}$ & Stérilité des femmes & $\begin{array}{l}\text { XXVIII, } \\
27\end{array}$ \\
\hline
\end{tabular}




\begin{tabular}{|l|l|l|}
\hline Graisse en fumigation & Chasse les serpents & $\begin{array}{l}\text { XXVIII, } \\
27\end{array}$ \\
\hline $\begin{array}{l}\text { Organes sexuels et } \\
\text { miel }\end{array}$ & $\begin{array}{l}\text { Aphrodisiaque (même pour les hommes qui répugnent au } \\
\text { commerce des femmes) }\end{array}$ & $\begin{array}{l}\text { XXVIII, } \\
27\end{array}$ \\
\hline Patte droite & Facilite l'accouchement (mais la gauche est mortelle!) & $\begin{array}{l}\text { XXVIII, } \\
27\end{array}$ \\
\hline $\begin{array}{l}\text { Anus attaché au bras } \\
\text { gauche }\end{array}$ & Philtre pour attirer les femmes & $\begin{array}{l}\text { XXVIII, } \\
27\end{array}$ \\
\hline $\begin{array}{l}\text { Vessie et vin } \\
\text { Contre l'incontinence d'urine }\end{array}$ & $\begin{array}{l}\text { XXVIII, } \\
27\end{array}$ \\
\hline
\end{tabular}

19 La hyène offre à Pline de nombreuses médications. Les organes sexuels de la hyène sont considérés comme un puissant aphrodisiaque. Cela repose sur la croyance antique de son changement de sexe. On la considérait en effet comme hermaphrodite. Pour Esope « on dit des hyènes qu'elles changent chaque année de nature, se convertissant une fois en mâle, une autre fois en femelle ${ }^{54} »$. Pourtant Aristote avait fermement dénoncé cette légende: «Ce qu'on raconte de ses organes génitaux, à savoir que le même individu posséderait à la fois les organes du mâle et de la femelle, est contraire à la véritéf5 ». Pline se fait l'écho des réserves d'Aristote: «Ordinairement, la croyance est que les hyènes possèdent deux sexes et que chaque année elles sont alternativement mâle ou femelle; elles engendrent sans mâle. Mais Aristote nie tout ceci ${ }^{56} »$. Malgré le déni d'Aristote, Pline reconnaît à ses organes sexuels leur puissance aphrodisiaque, même pour les hommes qui répugnent au commerce des femmes, hermaphrodisme oblige! Elien de Préneste, très friand de ce genre de récit, en oublie la critique aristotélicienne : « Tu peux voir comment une hyène, mâle cette année, se transformera en femelle l'an prochain. Elles participent des deux sexes et alternent la possibilité d'être mari ou femme, changeant chaque année, tellement bien que cet animal sans attitudes arrogantes mais avec des faits véridiques a relégué au rang de mythe Céné et Tirésia ${ }^{57}$ ». L'hermaphrodisme est devenu une vérité et si le Moyen Age ne devait pas mettre en doute cette croyance, pas question pour les clercs avec leur phobie de la sexualité de recommander la consommation de la partie honteuse de la hyène. L'animal est devenu le symbole de la duplicité. Pour le Physiologus « c'est un animal immonde ${ }^{58}$ ». Pierre de Beauvais précise dans son Bestiaire que "c'est une bête répugnante. Cette bête ressemble aux fils d'Israël qui, au commencement servirent Dieu, et s'adonnèrent ensuite aux délices du monde et à la luxure, et rendirent un culte aux idoles; et c'est pour cette raison que le Prophète affirme que la religion des juifs ressemble à cette bête ignoble. Toi chrétien, quel que tu sois, si tu as en toi l'amour des richesses, sache que la soumission à la cupidité est la racine de tous les maux, ainsi que le dit l'Apôtre, qui déclare : Ceux qui sont tels sont semblables à cette bête répugnante, car ils ne sont ni hommes ni femmes, ni loyaux ni fourbes, mais ils sont de ceux dont Salomon a dit: C'est un homme au cœur double, qui n'est ni constant ni sincère dans toute sa conduite, tout comme l'hyène qui se comporte à la fois en mâle et en femelle. A ces gens là, Notre-Seigneur a dit : Vous ne pouvez servir à la fois Dieu et le Diable ${ }^{59}$ ». La dualité la cohabitation du masculin et du féminin, du fort et du faible a rejeté la hyène dans le camp du mal et du péché et ne pouvait en faire, aux yeux des clercs, un animal thérapeutique. 


\section{Une approche irréaliste}

\section{ELEPHANT}

\begin{tabular}{|l|l|l|}
\hline Sang & $\begin{array}{l}\text { Fluxions appelées } \\
\text { rhumatismes }\end{array}$ & $\begin{array}{l}\text { XXVIII, } \\
24\end{array}$ \\
\hline Sang & Maladies consomptives & $\begin{array}{l}\text { XXVIII, } \\
24\end{array}$ \\
\hline Raclures d'ivoire et sciure & Panaris & $\begin{array}{l}\text { XXVIII, } \\
24\end{array}$ \\
\hline Raclures d'ivoire et miel & Taches de la face & $\begin{array}{l}\text { XXVIII, } \\
24\end{array}$ \\
\hline Toucher la trompe surtout si l'animal éternue & Maux de tête & $\begin{array}{l}\text { XXVIII, } \\
24\end{array}$ \\
\hline Partie droite de la trompe et terre rouge de & Aphrodisiaque & $\begin{array}{l}\text { XXVIII, } \\
24\end{array}$ \\
\hline Lemnos & & $\begin{array}{l}\text { XXVIII, } \\
24\end{array}$ \\
\hline Foie & Epilepsie & \\
\hline
\end{tabular}

\section{CHAMEAU}

\begin{tabular}{|l|l|l|}
\hline Cervelle desséchée et vinaigre (boisson) & Epilepsie & XXVIII, 26 \\
\hline Fiel et miel & Angines & XXVIII, 26 \\
\hline \hline Fiente en cendre et huile & Fait friser les cheveux & XXVIII, 26 \\
\hline Fiente en cendre (application) & Dysenterie & XXVIII, 26 \\
\hline Urine & Ulcères suintants, purgatif & XXVIII, 26 \\
\hline Soies de la queue nattées et attachées au bras gauche & Fièvre quarte & XXVIII, 26 \\
\hline
\end{tabular}

LION

\begin{tabular}{|l|l|l|}
\hline $\begin{array}{l}\text { Graisse et huile } \\
\text { rosat }\end{array}$ & $\begin{array}{l}\text { Taches du visage / Gonflements articulaires / Brûlures de la } \\
\text { neige }\end{array}$ & $\begin{array}{l}\text { XXVIII, } \\
25\end{array}$ \\
\hline Fiel et eau & Eclaircir la vue (en onction) & $\begin{array}{l}\text { XXVIII, } \\
25\end{array}$ \\
\hline
\end{tabular}




\begin{tabular}{|l|l|l|}
\hline Fiel et graisse & Epilepsie & $\begin{array}{l}\text { XXVIII, } \\
25\end{array}$ \\
\hline Cœur & Fièvre quarte & $\begin{array}{l}\text { XXVIII, } \\
25\end{array}$ \\
\hline Graisse en friction & Faire fuir les bêtes / Permet d'éviter les guet-apens & $\begin{array}{l}\text { XXVIII, } \\
25\end{array}$ \\
\hline
\end{tabular}

Pline donne de nombreuses médications utilisant des animaux difficilement accessibles (éléphant, lion, licorne...) ou des ingrédients rares et coûteux comme l'ivoire. Il fallait habiter la bonne région ou être riche pour se permettre de soigner ses rhumatismes avec du sang d'éléphant et un panaris, fut-il très douloureux, avec des raclures d'ivoire. Dans l'Occident du Haut Moyen Age, seul Charlemagne aurait pu guérir ses maux de tête en touchant la trompe d'un éléphant, grâce au pachyderme que lui avait envoyé jusqu'à Aixla-Chapelle Haroun al Raschid. Lorsque Hildegarde de Bingen, et bien d'autres avant elles, reprend sans sourciller ces recettes, elle ne semble pas se soucier de la difficulté à trouver les ingrédients nécessaires. L'abbesse commence dans son Livre des subtilités et des créatures divines, le chapitre consacré aux animaux terrestres par l'éléphant, le chameau et le lion avant même les animaux plus familiers, comme l'ours qui arrive en quatrième position. Elle recommande ainsi l'usage des os d'éléphant car c'est un animal qui contient la chaleur du soleil et donc une abondante sueur qui cuit les os et qui sont donc bons pour le rhume (refroidissement) - opposition traditionnelle du froid et du chaud -, la jaunisse ou les poumons. C'est pour l'abbesse un «bon » animal car sa chaleur vient du soleil et non de la chair, «il a plus d'os que de chair, pour ne pas tomber dans le dévergondage, car la chair aboutit toujours au dévergondage $\aleph^{60}$. Cette vision favorable de l'éléphant a été préparée par Aristote qui avait évoqué la chasteté du mâle qui ne s'approche plus de sa femelle après l'avoir fécondée ${ }^{61}$ et par Pline, louant sa piété au moment de la nouvelle lune où il se purifie dans un fleuve ${ }^{62}$. Elle prend bien soin de distinguer les trois (!) bosses du chameau. Elles détiennent respectivement la force du lion, du léopard et du cheval. L'os de la première, râpé dans de l'eau, soigne les douleurs, la deuxième les maux de rate, la troisième la gale et les fièvres ${ }^{63}$. De même, une femme qui ne parvient pas à accoucher doit «mettre un cœur de lion sur son nombril, pendant un temps assez bref, pas trop long : l'enfant se détachera en elle et arrivera aussitôt $\aleph^{64}$. Pour guérir un sourd, il faut lui appliquer, mais pas très longtemps, l'oreille droite d'un lion en prononçant ces paroles: «Entends, par le Dieu vivant et par la force pénétrante de l'ouïe du lion». Signalons en passant que le remède de la religieuse se démarque bien peu des pratiques païennes dénoncées par les conciles ou les livres pénitentiels ${ }^{65}$. Liant le geste à la parole, la vertu prêtée à l'animal est transférée par contact avec la partie malade en prononçant une formule de guérison qui ne se distingue des incantations des mages que par l'invocation de Dieu. La frontière entre la magie et la médecine « religieuse » est bien floue.

21 La guérison se produit grâce à la force du lion qui passe de son cœur, de son oreille ou de sa peau dans la personne malade. C'est le rayonnement de la chaleur léonine (« Le lion est totalement chaud » dit Hildegarde) qui pénètre l'homme et le guérit. A l'inverse, le cœur du tigre, placé encore chaud, sur la plaie récente d'un lépreux absorbe le mal. Mais si la lèpre est ancienne, cela ne fera qu'aggraver la maladie en la faisant pénétrer dans la chair 
de l'homme et en provoquant la décomposition de son cœur ${ }^{66}$. L'usage médical de ces animaux (lion ou tigre) est périlleux en raison de la force qui les habite et incite à la prudence.

De toute façon, les lecteurs de la Medicina Plinii ou de l'abbesse allemande devaient avoir beaucoup de mal à trouver en Occident et plus particulièrement dans la région de Bingen des os d'éléphant, des bosses de chameau ou un cœur de lion encore chaud. Cela montre une fois de plus le caractère livresque de cette science médicale, complètement déconnectée de la réalité, la grande dépendance des auteurs médiévaux vis-à-vis d'une faune scientifique héritée et un goût pour l'exotisme qui accorde la première place aux animaux lointains et fabuleux.

Mais ce merveilleux médical n'est pas incompatible avec des recettes plus réalistes et des ingrédients plus accessibles pour les malades (en particulier les plantes). La médecine de Pline offre à l'Occident médiéval un très grand choix d'animaux et de composants autochtones : sanglier, ours, cerf, lièvre, renard, blaireau, chevreau, taupe, musaraigne, lézard... Tout le règne animal est requis, y compris les volatiles et les bestioles les plus humbles mais aussi les plus répugnantes : vers de terre, mouches, punaises, mille-pattes...

On les retrouve en grande partie chez Hildegarde qui malgré tout manifeste de l'intérêt pour ces animaux autochtones qu'elle appelle souvent par leur nom germanique et non plus latin : rech (chevreuil), steinbock (bouquetin), wisant (bison), other (loutre), luchs (lynx), dash (blaireau), zobel (zibeline), waser marth (vison)... Il s'agit là d'une approche nouvelle et originale qui ne prévalait pas au Haut Moyen Age tant celui-ci se montrait dépendant face au savoir antique et méfiant vis-à-vis des pratiques populaires.

\section{Culture savante et culture populaire}

Il est souvent difficile d'établir une filiation précise entre le vaste savoir médical de Pline (avec ses centaines de médications) et la culture savante qui, à travers les encyclopédistes et autres compilateurs (d'Isidore de Séville à Hildegarde de Bingen...), l'a adapté, christianisé et complété par d'autres influences, y compris populaires. Toutes les pratiques quotidiennes, faisant appel à la magie, ont été condamnées par le clergé séculier (prêtres, évêques...) qui y voyaient l'expression de comportements païens qu'il fallait éradiquer avec la fermeté la plus grande. Or, certaines de ces médications populaires dont on a de très rares mentions tant la répression a été forte, présentent des similitudes avec les recommandations médicales de Pline ou d'ecclésiastiques du Haut Moyen Age. Elles semblent ainsi appartenir à un même fond de croyances, collectées en son temps par Pline et transmises ainsi par la culture savante, et en même temps, colportées de génération en génération par des individus qui ne connaissaient même pas l'existence du Naturaliste. On peut évoquer un fond commun que certains qualifieront d'indo-européen. Si autour de ces pratiques médicales on peut reconnaître d'indéniables liens entre la culture savante et la culture populaire, il n'en demeure pas moins que le Haut Moyen Age a fait une très nette distinction entre l'héritage de Pline et des autres autorités, reçu sans véritable remise en cause, y compris les racontars les plus étonnants, et les comportements quotidiens qui s'appuyaient souvent sur les mêmes animaux ou les mêmes éléments de la médecine plinienne. Le cas de la taupe est exemplaire. Pline, une fois n'est pas coutume, se montre très critique sur les usages de l'animal souterrain: "Voici une preuve particulière des mensonges des mages : de tous les animaux c'est la taupe qu'ils admirent le plus, elle qui est en tant d'égards maltraitée par la nature, 
condamnée à une cécité perpétuelle, enfouie de plus dans d'autres ténèbres où elle est comme enterrée. Il n'est pas de viscères auxquels ils accordent plus de confiance, il n'est pas d'animal qu'ils regardent comme plus propre aux rites religieux, au point qu'à celui qui avalera un cœur de taupe frais et palpitant, ils promettent de connaître par divination le déroulement des événements futurs. Ils affirment qu'on guérit les maux de dents en attachant sur soi une dent arrachée à une taupe vivante. Quant à leurs autres allégations touchant cet animal, nous en parlerons en leur place; ce qu'on y rencontrera de plus vraisemblable, c'est que les taupes combattent les morsures de musaraignes puisque, comme nous l'avons dit, la terres prises au fond des ornières les combat également ${ }^{67}$ ». Ce passage est tout à fait révélateur de l'esprit de Pline, critiquant les «mensonges des mages ", racontés par le menu, et proposant des médications tout aussi farfelues (la terre contre les morsures de musaraignes). Il rappelle dans plusieurs passages de son Histoire naturelle la nature prêtée à la taupe par les Anciens : sa cécité, son ouïe très fine qui la fait fuir, sa clairvoyance (malgré sa vue défaillante), son rôle dans les pratiques divinatoires ${ }^{68} . .$. Il rapporte ailleurs, sans la moindre réserve, d'autres recettes pour lutter contre les scrofules: "On conseille (...) de la cendre de taupe dans du miel. D'autres appliquent un foie de taupe écrasé dans les mains et ne lavent la région qu'après trois jours. On affirme aussi qu'une patte droite de la taupe est un remède pour les scrofules. D'autres coupent la tête de l'animal, la broient avec de la terre qu'il soulève, puis en composent des pastilles qu'ils gardent dans une boîte d'étain et dont ils se servent pour toutes les tuméfactions ${ }^{69}$ ». Il ajoute encore: «Les mages enseignent en outre que les délirants retrouvent la raison quand on les asperge de sang de taupe ${ }^{70}$ ». Cet animal chthonien, mangeur de terre, un des quatre éléments avec l'eau, l'air et le feu ${ }^{71}$, fort apprécié pour ses qualités divinatoires, a été rejeté et infernalisé par les clercs du Moyen Age. Pour Raban Maur « la taupe qui est condamnée à une cécité perpétuelle signifie une idole aveugle, sourde et muette ou également ces idolâtres eux-mêmes qui se fourvoient dans les ténèbres de l'ignorance et de la bêtise ${ }^{72}$ ». La taupe désigne aussi pour lui les hérétiques, les faux chrétiens qui, privés de la vraie lumière, s'adonnent aux tâches terrestres. L'animal souterrain, devenu le symbole du pécheur aveuglé, n'était plus considéré favorablement au Haut Moyen Age et lorsque l'évêque de Paris, Ragnemod, trouvait, à la fin du VI e siècle, un imposteur avec un sac rempli de dents de taupe, d'os de souris, de griffes et de graisse d'ours, on se dépêche de jeter ces sortilèges dans la Seine ${ }^{73}$. Les dents de taupe faisaient parties de l'arsenal thérapeutique de Pline mais il était évident, pour l'évêque de Paris, qu'elles s'intégraient à des rites magiques qu'il devait rejeter catégoriquement. Il n'y a pas de place pour ces pratiques populaires, même cautionnées par l'autorité de Pline. Le Haut Moyen Age est donc une période de répression où on a tenté d'occulter toutes ces pratiques magiques, en particulier celles qui avaient une destination médicale. Le développement du culte des saints et du pèlerinage sur leur tombeau a constitué une alternative à ces pratiques. Le saint, grâce au pouvoir qui lui était conféré par Dieu, annulait l'efficience des recettes magiques constituées d'ossements d'animaux et d'herbes. Les reliques étaient offertes en remplacement des amulettes, et les prières des incantations. La médecine des sanctuaires utilisait dorénavant la poussière du tombeau du saint, l'huile qui éclairait le lieu sanctifié pour composer les breuvages et onguents qui devaient rendre la santé ou la vue. Cela ne veut pas dire que l'Eglise ait réussi à éradiquer dans la vie quotidienne toutes les pratiques magiques, loin de là. Mais elle y est suffisamment parvenue pour qu'au XII ${ }^{e}$ siècle Hildegarde de Bingen puisse réintroduire la taupe dans ses pratiques thérapeutiques: « La taupe est froide (...), elle rejette la terre qui est mauvaise, malsaine 
et inutile, et reste dans celle qui est bonne et saine [la clairvoyance]. Elle ne voit rien puisqu'elle n'est pas dans l'air, mais elle a en elle une grande faculté de connaissance ; elle sent et devine où elle doit aller. Elle mange de la terre ». Hildegarde rappelle ainsi les diverses caractéristiques de sa nature définie par les Anciens et en tire une application médicale conforme à Pline mais en insistant sur le comportement original de l'animal qui rejette la mauvaise terre : «Si on a de l'infection en soi ou des scrofules sur le corps, on fera cuire une taupe avec de l'eau et on la mangera ; ou on la pilera et on mangera cette bouillie comme on pourra, et l'intérieur du corps sera assaini, ainsi que les scrofules, si elles ne s'étaient pas encore ouvertes : en effet, de même que la taupe rejette la terre qui est mauvaise, de même elle fait rejeter tout ce qui est mauvais à l'intérieur du corps de l'homme. L'homme ainsi atteint pourra manger le foie de la taupe avec le reste du corps, mais il ne mangera ni le cœur ni les poumons ${ }^{74} »$. Hildegarde ne précise pas pourquoi mais c'est probablement à cause des vertus divinatoires du cœur de la taupe qu'elle se garde bien de révéler à ses lecteurs. Comme Pline, elle recommande le sang de taupe, dans une médication très complexe qui intègre aussi de la poudre de bec de canard et d'oie, de la terre rejetée par la taupe, afin de soigner le haut mal (l'épilepsie). Elle reprend cette recette dans son ouvrage, Les causes et les remèdes, en justifiant son usage car « la taupe, qui tantôt se cache tantôt se montre, et qui a l'habitude de creuser le sol, à un sang qui s'oppose à cette maladie qui tantôt est apparente, tantôt se cache ${ }^{75}$ ». La taupe réussit à regagner l'arsenal thérapeutique, malgré l'image négative que les clercs continuent à lui imposer: sa nature souterraine opposée au ciel, les ténèbres où elle évolue, son aveuglement. C'est en particulier par la similitude entre les symptômes de l'épilepsie et le comportement de l'animal que sa récupération a pu se faire.

\section{Les principes actifs des médications}

\section{Quelques remèdes et vertus attribuées aux animaux}

\begin{tabular}{|c|c|c|}
\hline \multirow[t]{5}{*}{ Remèdes féminins } & Manger des testicules de lièvre pour avoir un garçon & \multirow[t]{5}{*}{$\begin{array}{l}\text { Pline, HN XXVIII, } \\
77\end{array}$} \\
\hline & Manger un foetus de lièvre pour rendre la fécondité & \\
\hline & Lait de truie et vin miellé facilite l'accouchement & \\
\hline & Lait d'ânesse pour les seins douloureux & \\
\hline & $\begin{array}{l}\text { Boire de l'urine de bouc et du nard contre les penchants } \\
\text { amoureux }\end{array}$ & \\
\hline
\end{tabular}




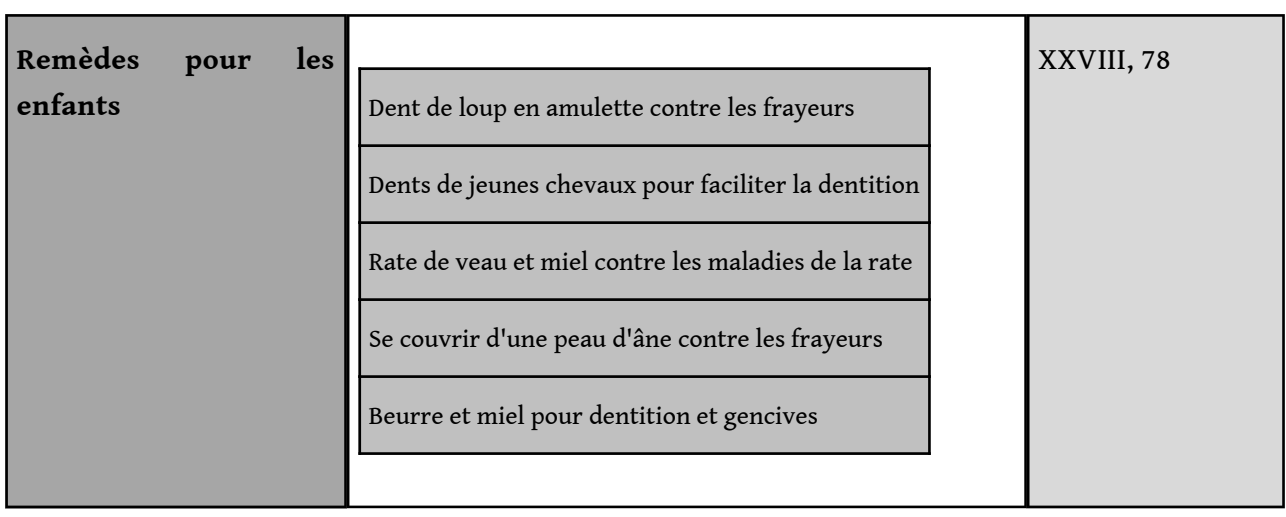

En effet, la science médicale médiévale repose sur quelques grands principes, déjà présents dans l'Antiquité et qui rendent les animaux relativement polyvalents dans leur utilisation, substituant tel animal par un autre parce qu'il est défaillant, introuvable (vivant dans la lointaine Afrique ou dans l'Orient merveilleux) ou peu recommandable à cause d'une nature peu conforme à la morale chrétienne (animal répugnant ${ }^{76}$ ). La nature d'un animal, les vertus qui lui sont attribuées influent certes fortement sur le choix de tel ou tel composant. L'idéal est de trouver une peau de lion ou un cœur de tigre mais on a recours en fait à des médicaments qu'on pourrait qualifier de "génériques " parce qu'ils s'appuient sur une famille d'animaux (les volatiles, les insectes, les animaux domestiqués) qui se distingue par un comportement, un point commun, une qualité qui va avoir une application thérapeutique. Ainsi le lion et le loup, malgré leur différence considérable de valorisation par les clercs, l'un figure le Christ et l'autre le Diable, se rejoignent sur leur nature de fauve prédateur. Pour Hildegarde " le loup est tout à fait chaud, et il a un peu (...) des mœurs du lion. (...) Grâce à sa nature de lion, il connaît et comprend l'homme et le flaire de $\operatorname{loin}^{77}$ ». L'eau de cuisson d'un loup, appliquée sur la tête des fous et des frénétiques, est tout aussi efficace que la peau de lion déposée sur le chef de ceux qui souffrent "de quelque faiblesse dans la tête ${ }^{78}$ », permettant ainsi de la réchauffer. La guérison est rendue possible grâce à la nature chaude du loup et du lion mais aussi à leur force que les auteurs antiques et médiévaux font résider dans la tête. Tous deux sont donc appropriés pour les maladies mentales même s'ils sont à manier avec la plus grande prudence (ne pas faire entrer l'eau de loup par les orifices et ne pas laisser en place trop longtemps la peau de lion).

Dans d'autres cas, plus que l'animal en personne, c'est une de ses parties qui constitue une sorte de concentré de la bête, ou qui met en évidence sa nature principale, qui est recherchée pour la médication. Ainsi, Pline recommande les dents de hyène, de loup pour soigner les douleurs dentaires parce que ces bêtes se caractérisent par leur agressivité dentaire. Mais n'importe quelle bête dont la gueule joue un rôle important dans son mode de vie peut être requise comme la taupe qui ronge et mange les racines ${ }^{79}$ mais aussi la couleuvre $^{80}$. Quant à l'utilisation de la dent de hyène pour combattre les peurs nocturnes, elle se fonde sur la nature de l'animal qui, selon le Naturaliste, en fait «la terreur particulière des panthères ${ }^{81} »$. La gueule et les crocs, capables de repousser d'aussi terribles fauves, deviennent autant de talismans contre les terreurs de la nuit. Leur efficacité repose sur cette fonction "mordicante » ou destructrice de la bête et peut s'appliquer à d'autres individus comme le loup (une dent portée en amulette protège efficacement les enfants de ce genre de désagrément) ${ }^{82}$. Cette conception médicale est donc régie par le principe de similitude: dent d'animal pour soigner les problèmes de 
dentition (dent de jeunes chevaux pour jeunes enfants), foie de loup ou d'âne pour les maladies hépatiques ${ }^{83}$, rate de bœuf avec du miel pour les maladies de la rate ${ }^{84}$, application de verge d'âne pour les défaillances sexuelles, vessie de sanglier pour les douleurs de vessie (vessie de truie pour les femmes) ${ }^{85} \ldots$ Les correspondances sont infinies: un fœetus de lièvre, animal réputé pour sa fertilité, assure la fécondité de la femme qui le mange; le lait de truie, bête connue pour sa capacité à mettre bas une abondante descendance, facilite l'accouchement; la consommation par la mère de testicules (de lièvre mais aussi d'autres animaux réputés pour leurs capacités génésiques) favorise la venue d'un garçon ${ }^{86} \ldots$ On retrouve le même principe de similitude chez les auteurs du Moyen Age et, en particulier, Hildegarde de Bingen : en cas de toux ou de manque de souffle, il faut manger du poumon de mouton; une femme dont la matrice est infertile consommera une matrice de brebis ou de génisse avant de s'accoupler avec son mari ${ }^{87} \ldots$

Ces médications reposent aussi sur le principe d'opposition : le chaud combat le froid, le sec l'humide, le masculin s'oppose au féminin, la droite à la gauche, la force à la faiblesse... La plupart des exemples que nous avons exposés y font référence.

Il faut en effet choisir avec précaution la partie appliquée pour le traitement (la partie droite de la trompe de l'éléphant, la patte droite de la hyène pour citer Pline, l'oreille droite du lion pour soigner la surdité chez Hildegarde) et ne pas se tromper sur le sexe de l'animal. Dans le cas de l'épilepsie, l'abbesse propose une médication faite de sang de taupe, de bec de canard femelle et de palmes d'oie femelle. Elle insiste sur ce point : « Ce bec et ces griffes doivent être ceux d'une femelle et non d'un mâle, à cause de son silence habituel, parce que la femelle est plus silencieuse que le mâle, de même que cette maladie demeure silencieuse jusqu'au moment où elle abat le malade ${ }^{88}$ ». Le jeu des oppositions se fonde aussi sur le couple extérieur-intérieur: la maladie qui se trouve à l'intérieur du malade peut sortir grâce au contact avec l'enveloppe d'un animal. La peau du haut de la tête d'un ours posée sur la poitrine et le cœur d'un individu timide, craintif, tremblant, anxieux le rendra, grâce à sa chaleur, énergique et sans peur ${ }^{89}$. La nature bénéfique de la bête est passée dans l'homme, à condition qu'il est pris soin de tanner avec précaution la peau pour enlever la sueur de la bête, qui, si elle rentrait en contact avec la chair de l'homme, pourrait susciter " une passion excessive » : désir charnel, violence bestiale. Le risque est toujours là de voir l'homme, en revêtant la peau de l'animal, se laisser investir par sa nature bestiale. La précaution dans l'utilisation de la peau d'ours constitue le fondement médical d'une croyance qui connaît un grand succès à partir du XII siècle, celle du loup-garou. Opposition, inversion, contraire participent donc à la démarche médicale.

Entre le $\mathrm{VI}^{\mathrm{e}}$ et le XII ${ }^{\mathrm{e}}$ siècle, d'Isidore de Séville à Hildegarde de Bingen, la pharmacopée antique, les conceptions médicales fondées sur les quatre éléments, les quatre humeurs se sont imposées avec force. Nous avons pu mesurer le poids de cet héritage antique, en particulier autour de la médication animale et des vertus prêtées à certains individus. L'originalité du Haut Moyen Age repose dans l'adaptation qui a été faite de cette matière médicale qu'il fallait parfois épurer de ses caractéristiques trop païennes. Sans remettre en cause l'héritage antique, les auteurs du Haut Moyen Age, pour l'essentiel des clercs, lui donne un éclairage chrétien qui renforce les vertus médicales de certains animaux comme le cerf, antique mangeur de serpents qui sait ensuite se purger du poison qui l'infecte, et dont l'abbesse Hildegarde recommande la consommation de sa chair comme purgatif ${ }^{90}$. Il y a donc une continuité très forte entre l'Antiquité et le Haut Moyen Age 
dans l'utilisation médicale de l'animal. Une rupture, encore timide, se produit à partir du XII ${ }^{\mathrm{e}}$ siècle, perceptible chez Hildegarde dans sa façon de décrire et d'envisager le monde animal. Mais c'est surtout à cette même époque en Orient que commence à se mettre en place une véritable critique de certaines recettes animales. C'est particulièrement le cas de Maimonnide (1135-1204), médecin juif de Saladin, qui décrit, dans son Traité des poisons, les animaux mais surtout dénonce les remèdes coûteux et inutiles utilisés pour servir de contrepoisons. Enfin, au XIII ${ }^{e}$ siècle, la redécouverte d'Aristote, débarrassé des scories de Pline, permet un renouveau de l'approche scientifique et accorde une place nouvelle à l'animal dans un contexte de désacralisation de la Nature.

\section{NOTES}

1. J'utilise en particulier les conclusions de mon chapitre II : l'héritage antique païen, une forte présence, Bestiaire chrétien, Toulouse, PUM, 1994, pp. 61-103.

2. Aymard J., "L'animal et les vertus romaines ", Hommages à Léon Hermann, Latomus, Bruxelles, 1960, XLIV, p. 123.

3. Pline, H.N., XXVIII, 30, 34. Fils du philosophe romain Quintus Sextius ( $\mathrm{I}^{\mathrm{er}} \mathrm{s}$. avant J.-C.), tous deux adeptes du néopythagorisme et fondateur de la «secte des Sextii » inspirée par un ascétisme végétarien.

4. Pline, H.N., XXVIII, 77.

5. Pline, H.N., XXVIII, 60, 215. Traduction de A. Ernout, Paris, Les Belles Lettres, 1962, réimpression 2003.

6. Pline, H.N., XXVIII, 8.

7. Pline, H.N., XXIX, 10, 28.

8. Pline, H.N., XXIX, 5, 11.

9. Pline, H.N., XXIX, 8, 17.

10. Pline, H.N., XXVIII, 77.

11. Pline, H.N., XXIX, 8, 18.

12. Elien, Nat. Anim., VI, 22 ; XIV, 9 ; III, 31.

13. Idem, IV, 29. Rappelons que le coq était l'oiseau d'Asclépios (Esculape), le dieu de la médecine, et du temple qui lui était consacré à Epidaure. Dans les représentations d'Asclépios, cet oiseau solaire (il est aussi l'attribut d'Hélios-Apollon) qui annonce le retour de l'astre et de la vie est souvent opposé au serpent, animal terrestre et froid. Quant au caducée médical, il est constitué d'un faisceau de baguettes autour duquel s'enroule le serpent d'Asclépios, surmonté du miroir de la prudence. Tardivement, le caducée a parfois présenté deux serpents affrontés (le caducée d'Hermès, les serpents étant alors considéré comme celui d'Asclépios et celui de sa fille Hygie (la santé).

14. Pline, H.N., XXVIII, XLII (149-151).

15. Isidore de Séville, Etymologiae XII, 1, 18, trad. J. André, Paris, Les Belles Lettres, 1986.

16. Physiologus Latinus, versio Y, XLIII, de cervo.

17. Raban Maur, De universo, VII, 8, Patrologie latine CXI, 204-205.

18. Psaume 42 (41), 1-2.

19. J. Voisenet, Bestiaire chrétien, Toulouse, PUM, 1994, pp. 309-310.

20. Félicie d'Ayzac, «Iconographie du cerf », Revue de l'art chrétien, 1884, VIII, p. 543. 
21. J. Voisenet, Bestiaire chrétien, Toulouse, 1994, p. 159.

22. Voir R. Delort, Les animaux ont une histoire, Paris, 1984, pp. 45-46.

23. Hildegarde de Bingen, le Livre des subtilités des créatures divines, Le livre des animaux, ch. X, trad. C. Mettra, Grenoble, Jérôme Million, 1994, p. 181.

24. Pline, H.N., XXVIII, 42 (10).

25. Hildegarde de Bingen, le Livre des subtilités des créatures divines, Le livre des animaux, ch. IX, trad. C. Mettra, Grenoble, 1994, p. 179.

26. Mat. 8, 30-31; Marc 5, 12 ; Luc 8, 33.

27. Vita s. Reguli, II, 7, Acta Sanctorum, éd. Bollandus, Mars III, 819. Voir J. Voisenet, Bêtes et hommes dans le monde médiéval, Turnhout, Brepols, 2000, p. 323.

28. Grégoire de Tours, De miraculis, I, 99.

29. Vita Patrum Iurensium, 51 (I, 16), trad. F. Martine, Sources chrétiennes, $\mathrm{n}^{\circ} 142$, Paris, 1968, p. 295.

30. Vita eparchii, I, 19, M.G.H. S.R.M. III, 559 : Non se carminibus sanum effici postulavit, sed ad suam orationem continuo sollicite accessit.

31. Elien, $\mathrm{V}, 29$.

32. Aristote, Histoire des animaux, VI, 18.

33. Aristote, La génération des animaux, IV, 5 .

34. Plaute, Cistel. 306 ; Cicéron, In Pisonem 69 ; Apulée, Métam., VI, 28 ; Ovide, Remedium amoris, 634 ; Claudien, Epithalames, 287-294...

35. Concilium Turonense, canon 42, M.G.H. Concilia, p. 292.

36. Burchard de Worms (965-1025), Corrector ou Medicus, tiré de son Decretorum Liber, Patrologie Latine de Migne, CXL, 949-1014.

37. Burchard, op. cit., Corrector, XX, 5, P.L. CXL, 974.

38. Hildegarde de Bingen, Le livre des subtilités..., Le livre des animaux, de l'âne.

39. On retrouve ici la théorie physiologique des quatre « humeurs » (le sang, la bile jaune, la bile noire, le flegme), transposition de la théorie physique des quatre éléments (terre, eau, air, feu).

40. Hildegarde, Le livre des subtilités..., Le livre des animaux, XVII : le porc.

41. Idem, chapitre XVII.

42. Hildegarde, Les causes et les remèdes (Causae et curae), 207, L'épilepsie, trad. P. Monat, Grenoble, Jérôme Million, 1997, p. 232.

43. Hildegarde, Idem, 182, La stérilité masculine, p. 207.

44. Hildegarde, Les causes et les remèdes, 194, Contre la luxure.

45. Hildegarde, idem, Du cheval. Cette digestion difficile est due autant à l'excès de chaleur de la viande de cheval qu'au fait qu'il ne soit pas un ruminant.

46. Voir J. Voisenet, Bestiaire chrétien, Toulouse, 1994, pp. 162-167.

47. Pline, H.N., XVIII, 24 (100). Voir aussi Horace, Carm. III, 4, 34.

48. Lettre 1 (de 732), M.G.H. Epistolae, III-1, 279.

49. Lettre 13 (de 751), M.G.H. Epistolae, III-1, 370.

50. Canones Gregorii, éd. F.W.H. Wasserschleben, Die Bussordnungen der abendlandischen Kirche, Halle, 1851, p. 160.

51. Confessional d'Egbert, Wasserschleben, idem, p. 300.

52. Hildegarde envisage trois péchés responsables de la lèpre : la goinfrerie et l'ivrognerie, la colère, la débauche. Chacun nécessite des médications animales différentes : crottes d'hirondelle, graisse de cigogne et de vautour pour le premier, sang de cheval pour le second, graisse d'oie et de poule, fumier de poule pour le troisième. Les causes et les remèdes, pp. 211-213.

53. Hildegarde, Le livre des subtilités..., XVII, le porc; Les causes et les remèdes, 185 : «Si Dieu ne veut pas qu'il soit guéri »...

54. Fable 242, La hyène et la renarde.

55. Histoire des animaux, VI, 32. 
56. Pline, H.N., VIII, 105 (44).

57. Elien, I, 25 : Céné, jeune fille changée en homme par Poséidon et Tirésias, le devin thébain, qui changea deux fois de sexe.

58. Physiologus Latinus, versio Y, XXXVII, de Yena hoc est belua.

59. Pierre de Beauvais, Bestiaire, des propriétés de l'hyène, trad. G. Bianciotto, Bestiaires du Moyen Age, Paris, 1980, p. 41.

60. Hildegarde, Le livre des subtilités..., Le livre des animaux, I, de l'éléphant.

61. Aristote, H.A., V, 14, $546 \mathrm{~b}$; IX, 46, 630 b.

62. Pline, H.N., VIII, I (1).

63. Hildegarde, Le livre des subtilités..., II, le chameau.

64. Hildegarde, Le livre des subtilités..., III, le lion.

65. Burchard de Worms, Corrector, LIV : «As-tu fais des envoûtements et des charmes comme le font les impies, tels que les porchers, les vachers et parfois les chasseurs quand ils récitent des incantations diaboliques? (...) Si oui : deux ans de jeûne ».

66. Hildegarde, Le livre..., Le livre des animaux, VI, le tigre.

67. Pline, H.N., XXX, VII, 19-20.

68. Pline, id., IX, 178 ; X, 191 ; XI, 139. Porphyre, De l'abstinence, II, 48 : « Ceux qui veulent recevoir en eux les âmes des animaux divinateurs avalent les organes essentiels de ces animaux, tel que le cœur des corbeaux, des taupes ou des faucons, et possèdent ainsi présente en eux l'âme de ces animaux, qui rend des oracles comme un dieu ». Trad. J. Bouffartigue, M. Patillon, Paris, Les Belles Lettres, 1979, p. 113.

69. Pline, H.N., XXX, 12 (38).

70. Pline, id., XXX, 24 (84).

71. On pensait, comme Richard de Fournival, qu'il existait quatre animaux qui se nourrissaient exclusivement de l'élément où ils évoluaient : la taupe de la terre, le hareng de l'eau, le pluvier de l'air et la salamandre du feu.

72. Raban Maur, De universo, VIII, II, Patrologie Latine CXI, 226-227.

73. Grégoire de Tours, Histoire des Francs, IX, VI.

74. Hildegarde, Le livre des subtilités..., Le livre des animaux, XXXVII, la taupe.

75. Hildegarde, Les causes et les remèdes, 206, l'épilepsie.

76. "Les animaux qui dévorent les autres, qui se nourrissent de nourritures corrompues et pullulent en faisant des petits, comme le loup, le chien, le cochon, sont, comme les herbes inutiles, impropres à l'alimentation ». Hildegarde, Le livre des subtilités..., préface au Livre des animaux.

77. Hildegarde, Le livre des subtilités..., le livre des animaux, XIX, le loup.

78. Hildegarde, idem, III, le lion.

79. Pline, H.N., XXX, 7, 20.

80. Pline, H.N., $\mathrm{XXX}, 8,26$.

81. Pline, H.N., XXVIII, 27, 93.

82. Pline, H.N., XXVIII, 78, 257.

83. Pline, H.N., XXVIII, $55,197$.

84. Pline, H.N., XXVIII, 78, 259.

85. Pline, H.N., XXVIII, 60, 212.

86. Tous ces animaux (le lièvre, la truie...) ont une connotation sexuelle marquée qui explique leur usage en liaison avec la fécondité. Le lièvre avait aussi, comme la hyène, la fâcheuse réputation de changer de sexe : «Il semble parfois changer de sexe, c'est-à-dire que le mâle fait parfois rentrer en lui ses parties génitales, si bien qu'il ressemble à une femelle, et parfois il ne les fait pas rentrer ». Hildegarde, Le livre des subtilités..., Le livre des animaux, XVIII, le lièvre.

87. Hildegarde, Le livre des subtilités..., Le livre des animaux, XV, le mouton.

88. Hildegarde, Les causes et les remèdes, 207. 
89. Hildegarde, Le livre des subtilités, Le livre des animaux, IV, l'ours.

90. Hildegarde, Le livre des subtilités, Le livre des animaux, X, Le cerf.

\section{AUTEUR}

\section{JACQUES VOISENET}

Toulouse 